
\title{
$\begin{array}{ll}\text { Research Square } & \text { They should not be considered conclusive, used to inform clinical practice, } \\ \text { or referenced by the media as validated information. }\end{array}$
}

\section{Synovial Fluid Neutrophils in Oligoarticular Juvenile Idiopathic Arthritis Have an Altered Phenotype and Impaired Effector Functions}

\author{
Sabine Arve-Butler \\ Lund University: Lunds Universitet \\ Tobias Schmidt \\ Lund University: Lunds Universitet \\ Anki Mossberg \\ Lund University: Lunds Universitet \\ Elisabet Berthold \\ Lund University: Lunds Universitet \\ Birgitta Gullstrand \\ Lund University: Lunds Universitet \\ Anders A Bengtsson \\ Lund University: Lunds Universitet \\ Fredrik Kahn \\ Lund University: Lunds Universitet \\ Robin Kahn ( $\square$ Robin.kahn@med.lu.se) \\ Lund University https://orcid.org/0000-0002-3167-1179
}

\section{Research article}

Keywords: juvenile idiopathic arthritis, neutrophil, synovial fluid, phenotype, phagocytosis, oxidative burst, reactive oxygen species

Posted Date: February 19th, 2021

DOI: https://doi.org/10.21203/rs.3.rs-200749/v1

License: (-) (1) This work is licensed under a Creative Commons Attribution 4.0 International License. Read Full License

Version of Record: A version of this preprint was published at Arthritis Research \& Therapy on April 9th, 2021. See the published version at https://doi.org/10.1186/s13075-021-02483-1. 


\section{Abstract}

\section{Background}

Neutrophils are the most prevalent immune cells in synovial fluid in inflamed joints of children with oligoarticular juvenile idiopathic arthritis (JIA). Despite this, little is known about neutrophil function at the site of inflammation in JIA and how local neutrophils contribute to disease pathogenesis. This study aimed to characterize phenotype and function of synovial fluid neutrophils in oligoarticular JIA.

\section{Methods}

Neutrophils obtained from paired blood and synovial fluid from patients with active oligoarticular JIA were investigated phenotypically ( $n=17)$ and functionally (phagocytosis and oxidative burst, $n=13$ ) by flow cytometry. In a subset of patients $(n=6)$, blood samples were also obtained during inactive disease at a follow-up visit. Presence of CD206-expressing neutrophils was investigated in synovial biopsies from four patients by immunofluorescence.

Results

Neutrophils in synovial fluid had an activated phenotype, characterized by increased CD66b and CD11b levels, and most neutrophils had a CD16 $\mathrm{CD} 2 \mathrm{~L}^{\text {low }}$ aged phenotype. A large proportion of the synovial fluid neutrophils expressed CD206, a mannose receptor not commonly expressed by neutrophils but by monocytes, macrophages and dendritic cells. CD206 expressing neutrophils were also found in synovial tissue biopsies. The synovial fluid neutrophil phenotype was not dependent on transmigration alone. Functionally, synovial fluid neutrophils had reduced phagocytic capacity and a trend towards impaired oxidative burst compared to blood neutrophils. In addition, the effector functions of the synovial fluid neutrophils correlated negatively with the proportion of $\mathrm{CD} 206^{+}$neutrophils.

\section{Conclusions}

Neutrophils in the inflamed joint in oligoarticular JIA were altered, both regarding phenotype and function. Neutrophils in the synovial fluid were activated, had an aged phenotype, had gained monocyte-like features and had impaired phagocytic capacity. The impairment in phagocytosis and oxidative burst was associated to the phenotype shift. We speculate that these neutrophil alterations might play a role in the sustained joint inflammation seen in JIA.

\section{Background}

Juvenile idiopathic arthritis (JIA), is an inflammatory rheumatic joint disease affecting children. Despite disease onset being at a young age, symptoms may be lifelong and include irreversible joint damage or growth disturbances $(1,2)$. The JIA diagnosis is an umbrella term including several subtypes with the common denominator of unexplained persistent arthritis occurring before the age of sixteen (3). The most common subtype in the western world is oligoarticular JIA (4), commonly characterized by asymmetric disease onset with inflammation in one to four large joints $(3,5)$.

Previously, the immunopathogenesis of oligoarticular JIA has been thought to be driven primarily by adaptive immune responses, as the disease is associated with HLA class II genetic variants and presence of autoantibodies (5). However, the importance of the innate immune system in oligoarticular JIA is becoming recognized, as abnormalities in the adaptive immune system cannot fully explain the pathology. This is reflected in the treatment of oligoarticular JIA, where commonly used drugs are either non-specific or target important components of the innate immune system such as tumor necrosis factor alpha (TNFa) and interleukin 6 (IL-6) $(6,7)$.

Neutrophils represent a major part of the innate immune system, and the pathology of several autoimmune rheumatic diseases are at least partly driven by dysfunctional neutrophils $(8,9)$. Neutrophils are the most common immune cell present in synovial fluid from inflamed joints in oligoarticular JIA, and studies of several JIA subtypes suggest that circulating neutrophils are activated $(6,10-12)$. It is reasonable to hypothesize that neutrophils are important also in oligoarticular JIA. However, neutrophils at the site of inflammation have rarely been studied in JIA, except for a recent study by Metzemaekers et al. which demonstrated that synovial fluid neutrophils in oligo- and polyarticular JIA have a distinct phenotype compared to circulating neutrophils (13).

The major neutrophil effector functions include phagocytosis and oxidative burst. Neutrophil phagocytosis is important not only as a defense against infection but also for clearance of potential autoantigens. Reactive oxygen species (ROS) were long considered harmful byproducts in sterile inflammation, but are now recognized for their immunosuppressive properties and impaired ROS production is associated with rheumatic disease both in patients and animal models of disease (8,14-20). Thus far, no studies have investigated synovial fluid neutrophil effector functions in oligoarticular JIA and their relation to the pathogenesis. 
The phenotype of synovial fluid neutrophils in JIA is insufficiently characterized, and two contradicting studies describe them as being highly activated (13) or resting (21). The study by Metzemaekers et al. further describes the synovial fluid neutrophils as hypersegmented and that a significant proportion of the neutrophils express markers not usually found on neutrophils, such as HLA-DR (13). Neutrophils with acquired traits of other myeloid cells, including antigen presenting capacity and expression of the mannose receptor CD206, have been described in other rheumatic diseases such as rheumatoid arthritis (RA) $(22,23)$ and adult-onset Still's disease (24).

In this study, we set out to investigate both phenotype and effector functions of neutrophils in paired blood and synovial fluid from children with oligoarticular JIA. To characterize several important aspects of neutrophil biology, we investigated neutrophil phenotype by surface marker expression related to activation, tissue migration, maturity and monocyte-like phenotype. Neutrophil function was analyzed in regard to both phagocytosis and ROS production. We hypothesized that synovial fluid neutrophils would differ from circulating neutrophils both regarding phenotype and function, and that the neutrophil alterations can be important in driving and maintaining the local joint inflammation in oligoarticular JIA.

\section{Methods}

\section{Study population}

Children fulfilling international league of associations for rheumatology (ILAR) criteria for persistent oligoarticular JIA, untreated or solely on nonsteroid anti-inflammatory drugs (NSAIDs) for at least six months, undergoing therapeutic arthrocentesis between 2016 and 2019 were included in the study, $n=17$. One patient (no 5) had received intraarticular steroids in another joint, two months prior to sampling. Synovial fluid was obtained from knee in all patients. Blood and synovial fluid during active arthritis were collected from 17 patients. Synovial tissue biopsies were obtained from four patients, one of which is not included in phenotype or function analyses due to ongoing treatment with methotrexate (patient no 18), in conjunction with synovial fluid aspiration. Follow-up blood samples from periods of inactive disease were collected from six of the patients. During periods of inactive disease some of the patients were treated with conventional or biologic disease-modifying antirheumatic drugs (DMARD). Patient characteristics are described in table 1. Twelve of the patients were also included in a previous study (25), indicated in table 1.

Healthy controls were included after informed consent. All controls were adults and contributed with blood and oral samples.

Preparation of synovial cells and cell-free synovial fluid

Synovial fluid was centrifuged at $400 \mathrm{~g}, 10 \mathrm{~min}$ to pellet the cells. Synovial cells were counted and resuspended in phosphate buffered saline (PBS) at a final concentration of $1 \times 10^{6} / \mathrm{ml}$ for use in flow cytometric staining and downstream purification procedures. The synovial fluid supernatant was transferred to a new tube and centrifuged a second time at $800 \mathrm{~g}, 10 \mathrm{~min}$ to pellet potential remaining cells or debris. The cellfree synovial fluid was collected and stored at $-80^{\circ} \mathrm{C}$.

\section{Neutrophil phenotyping and definitions}

Equal volumes of blood, synovial cell suspension or oral cell suspension were stained with antibody panels described in Supplemental figure 1. Whole blood samples were prepared after staining using TQ-prep with Immunoprep reagent system (Beckman Coulter) for red blood cell lysis and fixation of white cells. Synovial, oral and purified cell samples were washed with PBS after staining. Samples were analyzed on a FACS Canto II flow cytometer (BD Biosciences) or Cytoflex (Beckman Coulter) according to Supplemental figure 1. Kaluza software (Beckman Coulter) was used for data processing. Gating strategies are described in Supplemental figure 1.

Neutrophil maturity was determined using surface markers CD62L and CD16, where mature neutrophils are CD16 ${ }^{\text {hi }}$, CD62 ${ }^{\text {hi }}$, immature neutrophils are CD16 ${ }^{\mathrm{mid}}, \mathrm{CD} 62 \mathrm{~L}^{\text {hi }}$ and aged neutrophils are CD62 $\mathrm{L}^{\text {low }}(26-28)$. Activated neutrophils were defined as having high levels of $\mathrm{CD} 11 \mathrm{~b}$ and CD66b. High levels of CD14 and CD206 were considered features of macrophage/monocyte like phenotype.

\section{Immunofluorescence staining of synovial tissue biopsies}

Paraffin embedded biopsies in $3 \mathrm{~mm}$ sections mounted on microscope glass slides were baked for $1 \mathrm{~h}$ at $60{ }^{\circ} \mathrm{C}$ followed by deparaffinization in Neo-Clear (Merck) and a series of washes in ethanol of decreasing concentration. Antigen retrieval was performed in citrate buffer pH 6.0. Slides were blocked with $5 \%$ bovine serum albumin (BSA) and $5 \%$ normal goat serum (Abcam) for 30 min at room temperature prior to staining with primary antibodies rabbit anti-human MPO 1:200 (Dako A0398), and mouse anti-CD206 1:200 (Antibodies-Online clone:22-130) in PBS with 1\% BSA at $4{ }^{\circ} \mathrm{C}$ over night. After PBS washes, the slides were stained with secondary antibodies goat anti-rabbit Alexa Fluor 488 (Invitrogen) and donkey anti-mouse Alexa Fluor 568 (Abcam). Slides were mounted with Prolong Gold AntiFade with DAPI (Invitrogen). Images were taken using a Zeiss fluorescence microscope and processed in Fiji software. 
Neutrophils were isolated from heparinized whole blood from healthy controls using Lymphoprep (Axis-Shield) density gradient centrifugation according to manufacturer's instructions followed by sedimentation of red blood cells in saline with 1,5\% dextran T500 (Pharmacosmos).

Neutrophils in supernatant were collected, pelleted and contaminating red blood cells lysed with sterile $\mathrm{H}_{2} \mathrm{O}$. The purified neutrophils, $1 \times 10^{6} / \mathrm{ml}$, were incubated in MCDB 131 medium (Gibco) with $20 \%$ synovial fluid or $20 \%$ serum from blood donor at $37^{\circ} \mathrm{C}$ for $1 \mathrm{~h}$. Following incubation, neutrophils were washed with PBS and phenotypically analyzed by flow cytometry.

\section{Preparation of neutrophils from healthy oral cavity}

Healthy volunteers performed a mouth rinse with $5 \mathrm{ml} 0.9 \% \mathrm{NaCl}$ for 30 seconds in the morning, prior to breakfast and toothbrushing. The collected rinse fluid was filtered through $10 \mathrm{~mm}$ syringe filcons (BD Biosciences) and centrifuged $300 \mathrm{~g}, 5 \mathrm{~min}$. The pellet was resuspended in PBS with $0.1 \%$ BSA and 2 mM EDTA. Epithelial cells were removed by incubation of biotinylated anti-E-cadherin (Bioss) at a final concentration of 4 $\mathrm{mg} / \mathrm{ml}$ for 30 minutes followed by streptavidin-Dynabeads M280 (Invitrogen) and removal by magnet. The remaining cells were washed with PBS/BSA/EDTA. Leukocytes were isolated using DSB-X ${ }^{\mathrm{TM}}$ biotin labelled anti-CD 45 at $2 \mathrm{mg} / \mathrm{ml}$ (Dynabeads FlowComp Flexi kit, Invitrogen) and collected with Dynabeads FlowComp ${ }^{\mathrm{TM}}$ beads on a magnet. Cells were released from the beads using FlowComp ${ }^{\mathrm{TM}}$ release buffer, pelleted and resuspended in PBS.

\section{Neutrophil effector functions}

Phagocytosis and oxidative burst were analyzed using Phagoburst ${ }^{\mathrm{TM}}$ and Phagotest ${ }^{\mathrm{TM}}$ assay kit (BD Biosciences) according to manufacturer's instructions. Both methods are flow cytometric assays performed on $100 \mathrm{ml}$ heparinized whole blood or synovial fluid, measuring ROS production upon stimulation with phorbol-myristate-acetate (PMA) or opsonized E. coli and phagocytosis of fluorescently labelled opsonized E. coli respectively. When indicated, $90 \mathrm{ml}$ serum or synovial fluid was added to $10 \mathrm{ml}$ whole blood before analysis.

\section{Statistics}

Paired samples were analyzed using Wilcoxon's matched-pairs signed-rank test or paired t-test when indicated. Comparisons between groups of independent data were analyzed using Mann-Whitney U-test. Correlations were analyzed using Pearson r correlation coefficient. Statistical analyses were performed in Prism 9.

\section{Results}

\section{Description of the study population}

A total of 17 patients with active oligoarticular JIA, undergoing therapeutical synovial fluid aspiration, were included in the study. The patient cohort and samples are described in Table 1.

\section{Synovial fluid neutrophils have an aged and activated phenotype}

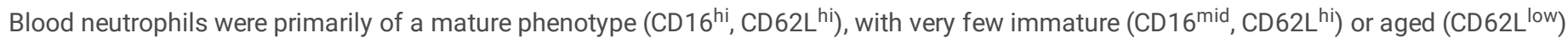
neutrophils. However, in synovial fluid, most neutrophils had an aged phenotype (range 41-99\%, median $76 \%$ ) and no immature neutrophils were observed (Figure 1A-D). Synovial fluid neutrophils also had higher levels of the age-related marker CD10 compared to neutrophils in the circulation (Figure 1E).

Neutrophils in synovial fluid had an activated phenotype, demonstrated by increased levels of activation markers CD11b and CD66b (Figure 1F$\mathrm{G}$ ), and low levels of CD62L, which is shed upon neutrophil activation and/or migration. Despite the activated phenotype, S100A8/A9, a protein released from activated neutrophils, was only present on the surface of less than $3 \%$ of both synovial fluid and blood neutrophils (Figure $1 \mathrm{H}$ ).

\section{Synovial fluid neutrophils express monocyte/macrophage-related surface markers}

Synovial fluid neutrophils had increased CD14 levels compared to the circulating neutrophils (Figure 1I). Mannose receptor CD206, not commonly known as a neutrophil receptor, but expressed by monocytes, macrophages and dendritic cells, was found on a significant proportion of synovial fluid neutrophils (range 6-92 \%, median $56 \%$ ), while being low or non-existent on circulating neutrophils (Figure $1 \mathrm{~J}$ ). The increased expression of both CD14 and CD206 might suggest that the neutrophils gain a monocyte/macrophage related phenotype.

\section{CD206-expressing neutrophils are found in tissue biopsies}

To confirm the finding of $\mathrm{CD}_{206^{+}}$neutrophils in synovial fluid we investigated whether neutrophils migrating through synovial tissue express CD206. Synovial tissue biopsies obtained from four patients were stained for CD206 and myeloperoxidase (MPO). Neutrophils co-expressing both MPO and CD206 were observed in tissue biopsies from patient 4, 8 and 18 (Figure 2, arrows), demonstrating that CD206-expressing neutrophils can be present in synovial tissue. In all biopsies there were also neutrophils without CD206 expression (Figure 2, arrow heads). In two of biopsies 
we observed synovial blood vessels, and the circulating neutrophils within the vessels had no or low CD206 expression (Figure 2B-C). The biopsy from patient 17 did not contain any area with neutrophils (data not shown).

\section{Circulating neutrophils are similar during inactive disease and flares}

Patients included in the study did not exhibit systemic symptoms although previous literature suggest that circulating neutrophils in JIA are activated during flares $(10,12,29)$. We therefore compared blood neutrophil phenotypes during flares and inactive disease. We did not observe systemic activation of neutrophils during flares; neutrophil activation markers CD66b and CD11b were mostly unchanged, CD62L levels slightly increased and cell surface S100A8/A9 was present on less than $3 \%$ of the neutrophils (Figure 3A-D). CD14 levels were markedly increased during flares (Figure 3E), while levels of CD16 and CD10 were not significantly altered (Figure 3F-G). CD206 expression, which was low or non-existent on blood neutrophils during flares, was equally low during inactive disease (Figure $3 \mathrm{H}$ ).

\section{The synovial neutrophil phenotype is not dependent on transmigration or synovial fluid alone}

To investigate if the synovial fluid neutrophil phenotype found in the JIA patients could be explained by exposure to synovial fluid, healthy blood neutrophils were treated with synovial fluid in vitro. Exposure to synovial fluid did not induce any major phenotype shift on healthy blood neutrophils (Figure 4A). Most surface markers remained unchanged except CD11b, which, in opposite to the findings in patients, was significantly lower after exposure to synovial fluid. There was a very modest increase of CD206 (Figure 4A). These results suggests that the phenotype shift between circulating and synovial fluid neutrophils cannot be explained by exposure to synovial fluid alone.

To investigate if tissue transmigration towards other sites would result in a phenotype shift similar to synovial fluid neutrophils, we investigated transmigrated neutrophils from the oral cavity of healthy controls. The phenotype shifts between healthy blood-and oral cavity neutrophils were compared with JIA blood- and synovial fluid neutrophils. Neutrophils in both oral cavity and joint were activated compared to their blood counterparts (Figure 4B-C). CD11b surface expression was elevated in the transmigrated neutrophils at both sites but more pronounced in synovial fluid compared to oral neutrophils (Figure 4C). On the other hand, neutrophil surface S100A8/A9 was markedly increased on oral cavity neutrophils but not on synovial fluid neutrophils (Figure 4D). Levels of CD62L were lower in oral neutrophils than synovial fluid neutrophils, compared to their circulating counterparts (Figure 4E). Oral neutrophils had dramatically decreased levels of CD16 while synovial fluid neutrophils had a small increase in this marker (Figure 4F). Both oral and synovial fluid neutrophils had increased levels of CD14 and CD206 (Figure 4G-H). CD10 levels were more increased in synovial compared to oral neutrophils, despite not reaching statistical significance (Figure 4I). Taken together, transmigration towards both sites induced a shift in neutrophil phenotypes, but with distinctly different surface marker patterns.

\section{Synovial fluid neutrophils have impaired phagocytosis and oxidative burst}

Neutrophil effector functions, phagocytosis and oxidative burst, were evaluated in blood and synovial fluid. Synovial fluid neutrophils had a significantly decreased ability to phagocytose opsonized E. coli compared to circulating neutrophils (Figure 5A). The impaired phagocytosis was not due to the presence of synovial fluid in the assay. Dilution of healthy control blood in cell-free JIA synovial fluid led to an increase in neutrophil phagocytic ability, in contrast to addition of JIA serum which led to a slight impairment in phagocytosis (Figure 5B). Upon stimulation with PMA, there was a trend towards impaired ROS production in synovial fluid neutrophils compared to circulating neutrophils, although not reaching statistical significance (Figure 5C). ROS production upon stimulation with opsonized E. coli was similar in synovial and circulating neutrophils (Figure 5D). Neutrophil capacity of phagocytosis and oxidative burst was not influenced by patient age (Supplemental Figure 2).

\section{Proportion of $\mathrm{CD}^{2} \mathrm{6}^{+}$neutrophils correlates with impaired phagocytosis and oxidative burst}

Monocytes typically have lower capacity for both phagocytosis and oxidative burst compared to neutrophils, and we therefore hypothesized that CD206 expression on synovial fluid neutrophils might be associated with altered effector functions. Indeed, we observed a negative correlation of the proportion of $\mathrm{CD}^{2} \mathrm{6}^{+}$neutrophils with both phagocytosis of opsonized E. coli (Figure 5E) and ROS production (Figure 5F-G) in synovial fluid neutrophils, supporting the hypothesis that synovial fluid neutrophils have attained a more monocyte-like phenotype.

\section{Discussion}

In this study, we show that synovial fluid neutrophils in active oligoarticular JIA have an activated, aged and transmigrated phenotype. The cells also have monocyte-like surface marker expression both in the synovial fluid and on migrating neutrophils within the synovial membrane. This monocyte-like phenotype is associated with reduced phagocytic capacity and ROS production. Thus, we suggest that neutrophils with altered functions are driving or sustaining the local inflammation in the joint of children with oligoarticular JIA.

In a healthy joint, the synovial fluid normally contains no or few immune cells. During arthritis, immune cells are recruited from the bloodstream by chemotactic stimuli. Neutrophils found in synovial fluid have extravasated from the synovial blood vessels and migrated through the synovial tissue that contains both synovial fibroblasts and infiltrating immune cells. In our study, the majority of these transmigrated neutrophils in synovial fluid had an aged phenotype, characterized by low surface levels of L-selectin (CD62L) and increased CD10 compared to neutrophils in 
the blood. The high amount of neutrophils with an aged phenotype in the joint could be due to anti-apoptotic effects of synovial fluid and inflammatory stimulation $(30,31)$. The presence of neutrophils with an aged phenotype in synovial fluid are supported by a recent study on synovial fluid neutrophils in oligo- and polyarticular JIA, which described the majority of synovial fluid neutrophils to be of a CD62L low and hypersegmented phenotype (13). However, CD62L is also shed upon neutrophil activation and migration (32). Thus, the CD62L low neutrophils seen in synovial fluid could be a consequence of both ageing and/or activation and tissue migration. Neutrophils may become activated by a wide variety of stimuli, such as inflammatory cytokines and chemotactic stimulation (33), and activated neutrophils present at a site of inflammation is expected. Indeed, synovial fluid neutrophils were highly activated, measured as increased levels of CD11b and CD66b. Nevertheless, neutrophils in the non-inflammatory oral cavity also had an increase in CD11b and CD66b, as these adhesion molecules are increased upon tissue migration. The activated phenotype of synovial fluid neutrophils is probably a consequence of combined inflammatory stimulation and tissue migration.

A large proportion of the synovial fluid neutrophils expressed the mannose receptor CD206, a receptor usually expressed by monocytes, macrophages and dendritic cells, which is upregulated on synovial fluid monocytes in JIA (25). To our knowledge, only one study has described CD206 on human neutrophils. In this study of adult onset Still's disease the authors found that CD206 appeared on circulating neutrophils during flare but not during inactive disease (24). CD206 ${ }^{+}$neutrophils are also described in a few publications of mouse models of stroke and myocardial infarction (34-36). We confirmed the presence of $C D 206^{+}$neutrophils in synovial biopsies where we found that some, but not all, of the neutrophils scattered within the synovial tissue had expression of CD206. The CD206 mannose receptor recognizes mannosylated and sulfated sugars, and has a wide variety of functions including phagocytosis and promotion of antigen presentation (37). In macrophages, increased expression of CD206 is associated with the "M2" phenotype and anti-inflammatory functions (38), but the role of CD206 on neutrophils is unknown. It seems as if the synovial fluid neutrophils are gaining a phenotype with features of both the neutrophil and monocyte lineages, based on the high amount of $\mathrm{CD}^{2} \mathrm{6}^{+}$neutrophils and increased levels of the TLR4 co-receptor CD14. Neutrophils expressing surface markers related to other myeloid phenotypes have previously been found in synovial fluid in JIA and RA. In the recent study of JIA synovial fluid neutrophils by Metzemaekers et al., a significant portion of the synovial fluid neutrophils had gained expression of HLA-DR (13), and synovial fluid neutrophils from RA patients have also been found to have antigen-presenting abilities $(22,23)$. As CD206 is described to be involved in cross-presentation of antigens (37), it is possible that expression of this receptor mediates antigen-presenting capacity of the neutrophils. Neutrophil plasticity to transdifferentiate into cells of other myeloid lineages is well established in vitro (39-41), and we believe that the microenvironment in the inflamed joint is affecting neutrophils to acquire other myeloid traits.

The phenotype shift seen in synovial fluid neutrophils is not dependent on either synovial fluid or transmigration separately. In vitro exposure of JIA synovial fluid to healthy blood neutrophils did not cause a phenotypical shift. We further studied oral cavity neutrophils as a control for tissue migration towards a non-inflammatory site and found that neutrophils at both sites shared some common features (activation, loss of CD62 $\mathrm{L}$ and gain of CD14 and CD206). However, the extent of the alterations was different in the two locations and other changes occurred in only one of the sites (increase of CD10 in synovial fluid, increase of S100A8/A9 in oral cavity and decrease of CD16 in oral cavity). The findings of distinct phenotypes varying between synovial fluid and oral cavity is supported by previous publications studying neutrophils in synovial fluid compared to skin blister fluid or pleural effusions $(13,21)$. Our results indicate that the synovial neutrophil phenotype and function alterations are induced by a combination of multiple factors and stimuli, and further studies are needed to characterize this.

Importantly, we found a significant decrease in phagocytic capacity of the synovial fluid neutrophils compared to circulating neutrophils. In addition, the phenotype shift of synovial neutrophils towards a monocyte-like pattern with increased surface expression of CD206 was associated with both reduced capacity of phagocytosis and oxidative burst, suggesting that alterations in phenotype and function are connected. Impaired neutrophil function may drive inflammation and autoimmunity, as neutrophil clearance of debris and dead cell remnants is essential to maintain homeostasis and minimize the autoantigenic burden (8). Extracellular ROS produced by neutrophils are also important for immunoregulation $(17,42)$ and impaired neutrophil ROS production in the joint could prolong the resolution of inflammation. As we demonstrate that addition of synovial fluid to healthy control neutrophils does not impair phagocytic capacity, we suggest that the impairment in effector functions is an inherent trait of the synovial fluid neutrophils and not a consequence of the local microenvironment.

The neutrophil gating strategy for patient neutrophils, and the fact that more aspects of synovial fluid neutrophil effector functions were not investigated present some limitations to the study. The panels were designed for negative selection of neutrophils, but as synovial fluid may contain cells and particles with neutrophil size and granularity without leukocyte surface markers, a positive selection marker was also needed. After negative selection, neutrophils were therefore selected by CD16 positivity, which may inadvertently lose immature CD16 low neutrophils. Neutrophil function is not limited to oxidative burst and phagocytosis, and to further characterize the functional differences between circulating and synovial fluid neutrophils in JIA including chemotaxis, release of neutrophil extracellular traps (NETs) and acquired ability to present antigens would be of interest and importance. These possible functional alterations will be investigated in further studies.

\section{Conclusions}


This study demonstrates that neutrophils in synovial fluid of children with active oligoarticular JIA are altered both phenotypically and functionally compared to paired neutrophils from the circulation. We observed that synovial fluid neutrophils are activated, aged and has gained a monocyte-like phenotype, which is associated with an impairment in neutrophil phagocytosis and oxidative burst. We suggest that these neutrophil alterations could be of importance in sustaining joint inflammation and thus interesting possible targets for specific therapies.

\section{Abbreviations}

JIA - juvenile idiopathic arthritis, ROS - reactive oxygen species, PMA - phorbol 12-myristate 13-acetate, NSAID - non-steroid anti-inflammatory drug, MFI - median fluorescence intensity, RA - rheumatoid arthritis

\section{Declarations}

Ethics approval and consent to participate

Collection of JIA patient samples and data and healthy controls were approved by the Regional Ethical Review Board for southern Sweden ((LU 2015/734, LU2016/128 and LU2017/473). Informed consent was obtained from the healthy control, patient and/or the patient's guardian.

Consent for publication

Not applicable.

Availability of data and materials

Anonymized datasets used and/or analyzed during the current study are available from the corresponding author on reasonable request.

Competing interests

The authors declare no financial or competing interests.

Funding

This study was supported by grants from the Crown Princess Lovisa's Society for Child Care, the Swedish Rheumatism Association, Greta and Johan Kock's Foundation, the Hedberg Foundation, the Anna-Greta Crafoord Foundation, the Crafoord Foundation, the Swedish Medical Society, the Jerring Foundation, Alfred Österlunds Foundation, Magnus Bergvall Foundation, Thelma Zoega's foundation, Lion research foundation, King Gustaf V's 80-year foundation, The Knut and Alice Wallenberg foundation, the Medical Faculty at Lund University and Region Skåne (all to RK).

Authors' contributions

SAB carried out the experiments and data analysis, interpreted data, and wrote the manuscript.

TS, AM, BG carried out the experiments and reviewed and revised the manuscript.

EB collected clinical data and samples, as well as reviewed and revised the manuscript.

FK and $A A B$ interpreted data, reviewed and revised the manuscript.

RK conceptualized and designed the study, collected clinical data and samples, interpreted data, and wrote the manuscript.

All authors have read and approved the final manuscript.

Acknowledgements

We wish to acknowledge Åsa Pettersson for support in the phagotest and phagoburst experiments, and Daniel Butler for lending us secondary antibodies and microscope access for immunofluorescence.

\section{References}

1. Glerup M, Rypdal V, Arnstad ED, Ekelund M, Peltoniemi S, Aalto K, et al. Long-Term Outcomes in Juvenile Idiopathic Arthritis: Eighteen Years of Follow-Up in the Population-Based Nordic Juvenile Idiopathic Arthritis Cohort. Arthritis Care Res (Hoboken). 2020;72(4):507-16.

2. Matsumoto T, Matsui T, Hirano F, Tohma S, Mori M. Disease activity, treatment and long-term prognosis of adult juvenile idiopathic arthritis patients compared with rheumatoid arthritis patients. Mod Rheumatol. 2020 Jan;30(1):78-84.

3. Prakken B, Albani S, Martini A. Juvenile idiopathic arthritis. Lancet. 2011 Jun 18;377(9783):2138-49. 
4. Berthold E, Månsson B, Kahn R. Outcome in juvenile idiopathic arthritis: a population-based study from Sweden. Arthritis Res Ther. 2019 Oct 28;21(1):218.

5. Macaubas C, Nguyen K, Milojevic D, Park JL, Mellins ED. Oligoarticular and polyarticular JIA: epidemiology and pathogenesis. Nat Rev Rheumatol. 2009 Nov;5(11):616-26.

6. Jarvis JN, Jiang K, Petty HR, Centola M. Neutrophils: the forgotten cell in JIA disease pathogenesis. Pediatr Rheumatol Online J. 2007 Jun 13;5:13.

7. Giancane G, Alongi A, Ravelli A. Update on the pathogenesis and treatment of juvenile idiopathic arthritis. Curr Opin Rheumatol. 2017 Sep;29(5):523-9.

8. Wirestam L, Arve S, Linge P, Bengtsson AA. Neutrophils-Important Communicators in Systemic Lupus Erythematosus and Antiphospholipid Syndrome. Front Immunol. 2019 Nov 22;10:2734.

9. Wright HL, Moots RJ, Edwards SW. The multifactorial role of neutrophils in rheumatoid arthritis. Nat Rev Rheumatol. 2014 Oct;10(10):593601.

10. Jarvis JN, Petty HR, Tang Y, Frank MB, Tessier PA, Dozmorov I, et al. Evidence for chronic, peripheral activation of neutrophils in polyarticular juvenile rheumatoid arthritis. Arthritis Res Ther. 2006;8(5):R154.

11. Brown RA, Henderlight M, Do T, Yasin S, Grom AA, DeLay M, et al. Neutrophils From Children With Systemic Juvenile Idiopathic Arthritis Exhibit Persistent Proinflammatory Activation Despite Long-Standing Clinically Inactive Disease. Front Immunol. 2018 Dec 18;9:2995.

12. Ramanathan K, Glaser A, Lythgoe H, Ong J, Beresford MW, Midgley A, et al. Neutrophil activation signature in juvenile idiopathic arthritis indicates the presence of low-density granulocytes. Rheumatology. 2018 Mar 1;57(3):488-98.

13. Metzemaekers M, Malengier-Devlies B, Yu K, Vandendriessche S, Yserbyt J, Matthys P, et al. Synovial fluid neutrophils from patients with juvenile idiopathic arthritis display a hyperactivated phenotype. Arthritis Rheumatol. 2020 Dec 2;

14. Bengtsson AA, Pettersson Å, Wichert S, Gullstrand B, Hansson M, Hellmark T, et al. Low production of reactive oxygen species in granulocytes is associated with organ damage in systemic lupus erythematosus. Arthritis Res Ther. 2014 Jun 5;16(3):R120.

15. Olsson LM, Johansson ÅC, Gullstrand B, Jönsen A, Saevarsdottir S, Rönnblom L, et al. A single nucleotide polymorphism in the NCF1 gene leading to reduced oxidative burst is associated with systemic lupus erythematosus. Ann Rheum Dis. 2017 Jun 12;

16. Linge P, Arve S, Olsson LM, Leonard D, Sjöwall C, Frodlund M, et al. NCF1-339 polymorphism is associated with altered formation of neutrophil extracellular traps, high serum interferon activity and antiphospholipid syndrome in systemic lupus erythematosus. Ann Rheum Dis. 2020 Feb;79(2):254-61.

17. Hoffmann MH, Griffiths HR. The dual role of ROS in autoimmune and inflammatory diseases: Evidence from preclinical models. Free Radic Biol Med. 2018 Mar 15;125:62-71.

18. Urbonaviciute V, Luo H, Sjöwall C, Bengtsson A, Holmdahl R. Low production of reactive oxygen species drives systemic lupus erythematosus. Trends Mol Med. 2019 Jul 11;25(10):826-35.

19. Kelkka T, Kienhöfer D, Hoffmann M, Linja M, Wing K, Sareila O, et al. Reactive oxygen species deficiency induces autoimmunity with type 1 interferon signature. Antioxid Redox Signal. 2014 Dec 1;21(16):2231-45.

20. Zhao J, Ma J, Deng Y, Kelly JA, Kim K, Bang S-Y, et al. A missense variant in NCF1 is associated with susceptibility to multiple autoimmune diseases. Nat Genet. 2017 Mar;49(3):433-7.

21. Björkman L, Christenson K, Davidsson L, Mårtensson J, Amirbeagi F, Welin A, et al. Neutrophil recruitment to inflamed joints can occur without cellular priming. J Leukoc Biol. 2018 Dec 20;

22. Iking-Konert C, Ostendorf B, Sander O, Jost M, Wagner C, Joosten L, et al. Transdifferentiation of polymorphonuclear neutrophils to dendriticlike cells at the site of inflammation in rheumatoid arthritis: evidence for activation by T cells. Ann Rheum Dis. 2005 Oct;64(10):1436-42.

23. Cross A, Bucknall RC, Cassatella MA, Edwards SW, Moots RJ. Synovial fluid neutrophils transcribe and express class II major histocompatibility complex molecules in rheumatoid arthritis. Arthritis Rheum. 2003 Oct;48(10):2796-806.

24. Kim H-A, Choi B, Suh C-H, Han MH, Jung J-Y, Sayeed HM, et al. Highly Expression of CD11b and CD32 on Peripheral Blood Mononuclear Cells from Patients with Adult-Onset Still's Disease. Int J Mol Sci. 2017 Jan 19;18(1).

25. Schmidt T, Berthold E, Arve-Butler S, Gullstrand B, Mossberg A, Kahn F, et al. Children with oligoarticular juvenile idiopathic arthritis have skewed synovial monocyte polarization pattern with functional impairment-a distinct inflammatory pattern for oligoarticular juvenile arthritis. Arthritis Res Ther. 2020 Aug 12;22(1):186.

26. Grieshaber-Bouyer R, Nigrovic PA. Neutrophil Heterogeneity as Therapeutic Opportunity in Immune-Mediated Disease. Front Immunol. 2019 Mar 4;10:346.

27. van Grinsven E, Textor J, Hustin LSP, Wolf K, Koenderman L, Vrisekoop N. Immature Neutrophils Released in Acute Inflammation Exhibit Efficient Migration despite Incomplete Segmentation of the Nucleus. J Immunol. 2019 Jan 1;202(1):207-17. 
28. Pillay J, Kamp VM, van Hoffen E, Visser T, Tak T, Lammers J-W, et al. A subset of neutrophils in human systemic inflammation inhibits T cell responses through Mac-1. J Clin Invest. 2012 Jan;122(1):327-36.

29. Foell D, Wittkowski H, Hammerschmidt I, Wulffraat $\mathrm{N}$, Schmeling $\mathrm{H}$, Frosch $\mathrm{M}$, et al. Monitoring neutrophil activation in juvenile rheumatoid arthritis by S100A12 serum concentrations. Arthritis Rheum. 2004 Apr;50(4):1286-95.

30. Ottonello L, Cutolo M, Frumento G, Arduino N, Bertolotto M, Mancini M, et al. Synovial fluid from patients with rheumatoid arthritis inhibits neutrophil apoptosis: role of adenosine and proinflammatory cytokines. Rheumatology. 2002 Nov;41(11):1249-60.

31. Cross A, Barnes T, Bucknall RC, Edwards SW, Moots RJ. Neutrophil apoptosis in rheumatoid arthritis is regulated by local oxygen tensions within joints. J Leukoc Biol. 2006 Sep;80(3):521-8.

32. Ivetic A. A head-to-tail view of L-selectin and its impact on neutrophil behaviour. Cell Tissue Res. 2018 Jan 20;371(3):437-53.

33. Vogt KL, Summers C, Chilvers ER, Condliffe AM. Priming and de-priming of neutrophil responses in vitro and in vivo. Eur $\mathrm{J}$ Clin Invest. 2018 Nov;48 Suppl 2:e12967.

34. Cuartero MI, Ballesteros I, Moraga A, Nombela F, Vivancos J, Hamilton JA, et al. N2 neutrophils, novel players in brain inflammation after stroke: modulation by the PPARy agonist rosiglitazone. Stroke. 2013 Dec;44(12):3498-508.

35. Cai W, Liu S, Hu M, Huang F, Zhu Q, Qiu W, et al. Functional dynamics of neutrophils after ischemic stroke. Transl Stroke Res. 2020 Feb;11(1):108-21.

36. Ma Y, Yabluchanskiy A, lyer RP, Cannon PL, Flynn ER, Jung M, et al. Temporal neutrophil polarization following myocardial infarction. Cardiovasc Res. 2016 May 1;110(1):51-61.

37. Martinez-Pomares L. The mannose receptor. J Leukoc Biol. 2012 Dec;92(6):1177-86.

38. Mantovani A, Sica A, Sozzani S, Allavena P, Vecchi A, Locati M. The chemokine system in diverse forms of macrophage activation and polarization. Trends Immunol. 2004 Dec;25(12):677-86.

39. Araki H, Katayama N, Yamashita Y, Mano H, Fujieda A, Usui E, et al. Reprogramming of human postmitotic neutrophils into macrophages by growth factors. Blood. 2004 Apr 15;103(8):2973-80.

40. Köffel R, Meshcheryakova A, Warszawska J, Hennig A, Wagner K, Jörgl A, et al. Monocytic cell differentiation from band-stage neutrophils under inflammatory conditions via MKK6 activation. Blood. 2014 Oct 23;124(17):2713-24.

41. Takashima A, Yao Y. Neutrophil plasticity: acquisition of phenotype and functionality of antigen-presenting cell. J Leukoc Biol. 2015 Oct;98(4):489-96.

42. Hultqvist M, Olsson LM, Gelderman KA, Holmdahl R. The protective role of ROS in autoimmune disease. Trends Immunol. 2009 May;30(5):201-8.

\section{Tables}

Table I. Description of patient cohort. 


\begin{tabular}{|c|c|c|c|c|c|c|c|c|c|c|c|c|}
\hline Pat\# & Sex & $\begin{array}{l}\text { Disease } \\
\text { duration } \\
\text { (months) }\end{array}$ & $\begin{array}{l}\text { Age } \\
\text { (years) }\end{array}$ & Treatment & Uveitis & ANA & $\begin{array}{l}\text { Phenotype } \\
\text { analysis }\end{array}$ & $\begin{array}{l}\text { Functional } \\
\text { assay }\end{array}$ & Biopsy & $\begin{array}{l}\text { Follow- } \\
\text { up } \\
\text { sample }\end{array}$ & $\begin{array}{l}\text { Time to } \\
\text { follow- } \\
\text { up } \\
\text { (months) }\end{array}$ & $\begin{array}{l}\text { Treatment } \\
\text { at follow } \\
\text { up }\end{array}$ \\
\hline $1 *$ & $M$ & 132 & 15 & No & No & Pos & Yes & No & No & No & & \\
\hline 2 & $\mathrm{~F}$ & 26 & 7 & NSAID & No & Pos & Yes & No & No & No & & \\
\hline $3^{*}$ & $\mathrm{~F}$ & 88 & 11 & No & No & Pos & Yes & Yes & No & Yes & 40 & No \\
\hline $4^{*}$ & $\mathrm{~F}$ & 0 & 6 & NSAID & Yes & Pos & Yes & Yes & Yes & No & & \\
\hline $5^{\star}$ & $\mathrm{F}$ & 3 & 11 & NSAID & Yes & Pos & Yes & Yes & No & Yes & 34 & NSAID \\
\hline $6^{*}$ & $\mathrm{~F}$ & 49 & 7.5 & NSAID & No & Pos & Yes & No & No & Yes & 31 & TNFi \\
\hline $7 *$ & $\mathrm{~F}$ & 48 & 16.5 & No & Yes & Pos & Yes & Yes & No & No & & \\
\hline $8^{*}$ & $\mathrm{~F}$ & 2 & 3.5 & No & No & Pos & Yes & Yes & Yes & No & & \\
\hline $9 *$ & $\mathrm{~F}$ & 1 & 15 & NSAID & No & Pos & Yes & Yes & No & Yes & 20 & $\begin{array}{l}\text { Mtx, } \\
\text { NSAIID }\end{array}$ \\
\hline $10 *$ & M & 1 & 12 & NSAID & No & $\mathrm{Neg}$ & Yes & Yes & No & No & & \\
\hline 11 & $\mathrm{M}$ & 4 & 10 & NSAID & No & Pos & Yes & Yes & No & Yes & 19 & Mtx \\
\hline $12^{*}$ & $\mathrm{~F}$ & 0 & 2 & No & No & Pos & Yes & Yes & No & Yes & 16 & Mtx \\
\hline 13 & $\mathrm{~F}$ & 144 & 17 & NSAID & No & $\mathrm{Neg}$ & Yes & Yes & No & No & & \\
\hline $14^{\star}$ & $\mathrm{F}$ & 1 & 13 & NSAID & No & $\mathrm{Neg}$ & Yes & Yes & No & No & & \\
\hline 15 & $M$ & 2 & 4 & NSAID & No & Pos & Yes & No & No & No & & \\
\hline 16 & $M$ & 0 & 6 & NSAID & No & Pos & Yes & Yes & No & No & & \\
\hline $17 *$ & $\mathrm{~F}$ & 1 & 11 & No & No & $\mathrm{Neg}$ & Yes & Yes & Yes & No & & \\
\hline 18 & $\mathrm{~F}$ & 50 & 5 & Mtx & Yes & Pos & No & No & Yes & No & & \\
\hline
\end{tabular}

Clinical and sample data. Disease duration is calculated as months since date of diagnosis. Abbreviations: TNFi - TNF inhibitor, Mtx methotrexate.

* patients are also included in Schmidt et al. (25)

\section{Figures}



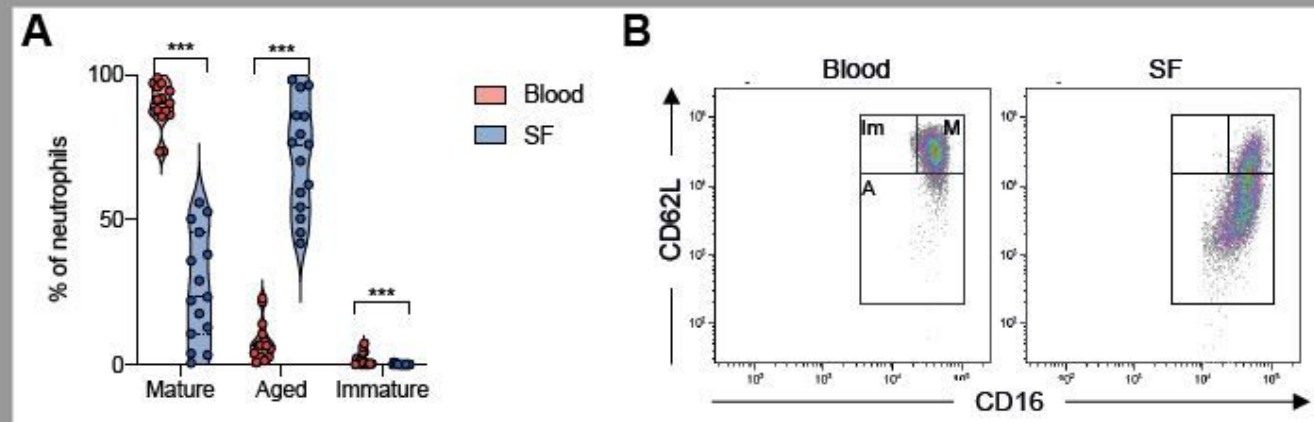

C

D

E

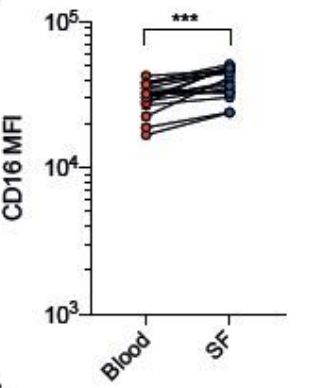

G
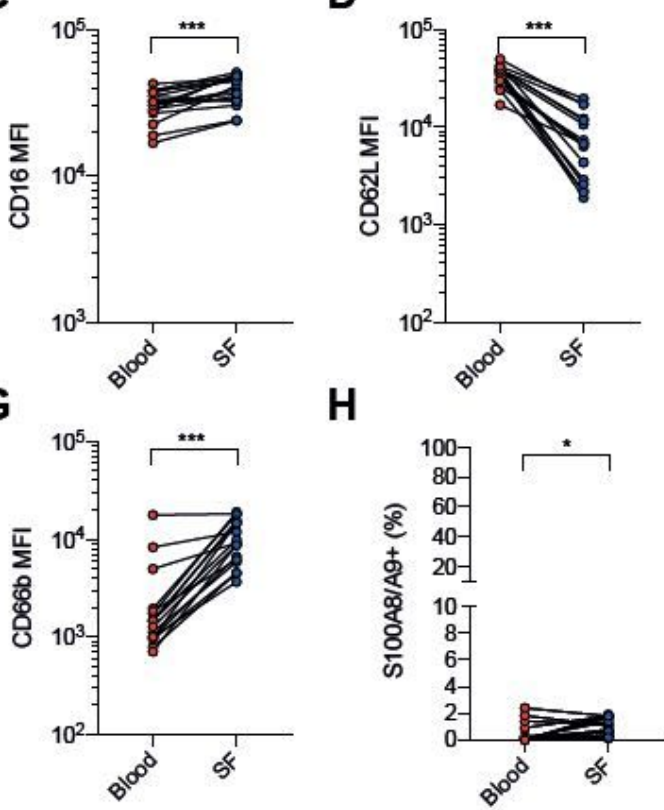

H
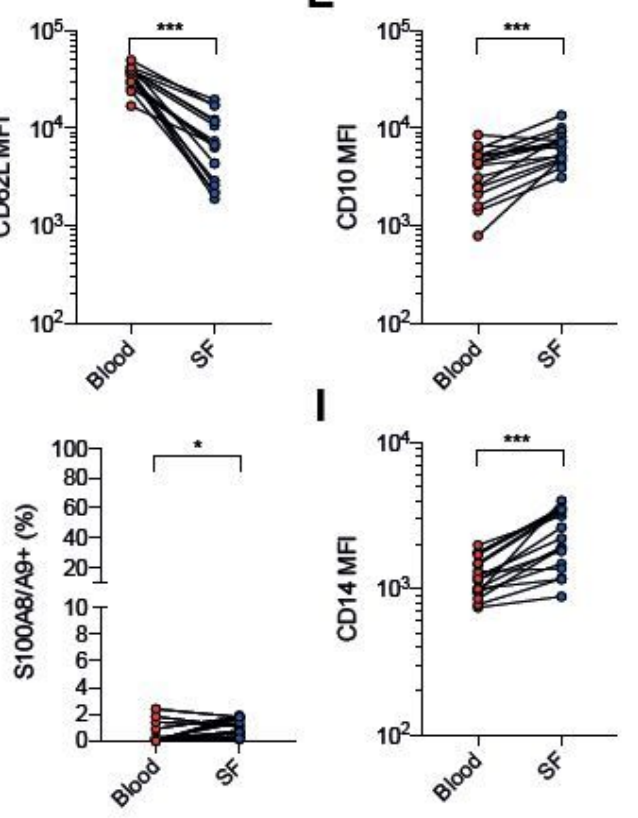

F

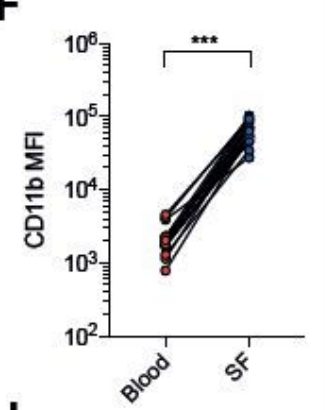

J

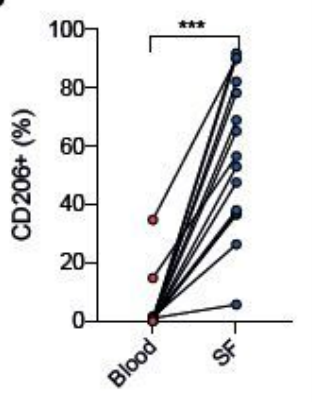

\section{Figure 1}

Synovial fluid neutrophils have an aged and activated phenotype and express monocyte related markers. Neutrophils from paired samples of blood and synovial fluid from patients with oligoarticular JIA were analyzed by flow cytometry. A) Neutrophilphenotypical maturities in blood and synovial fluid based on CD62L and CD16 expression. B) Representative plot of neutrophil maturity phenotype gating. Mature neutrophils (M) are CD16hi CD62Lhi, immature neutrophils ( $\mathrm{Im}$ ) are CD16mid CD62Lhi and neutrophils with decreased CD62L are CD62Llow (L). C-G \& I) Median fluorescence intensity (MFI) of neutrophil expression of C) CD16, D) CD62L, E) CD10, F) CD11b, G) CD66b and I) CD14. H \& J) Proportion of

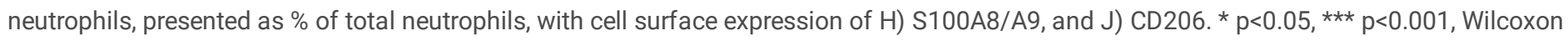
signed-rank test 


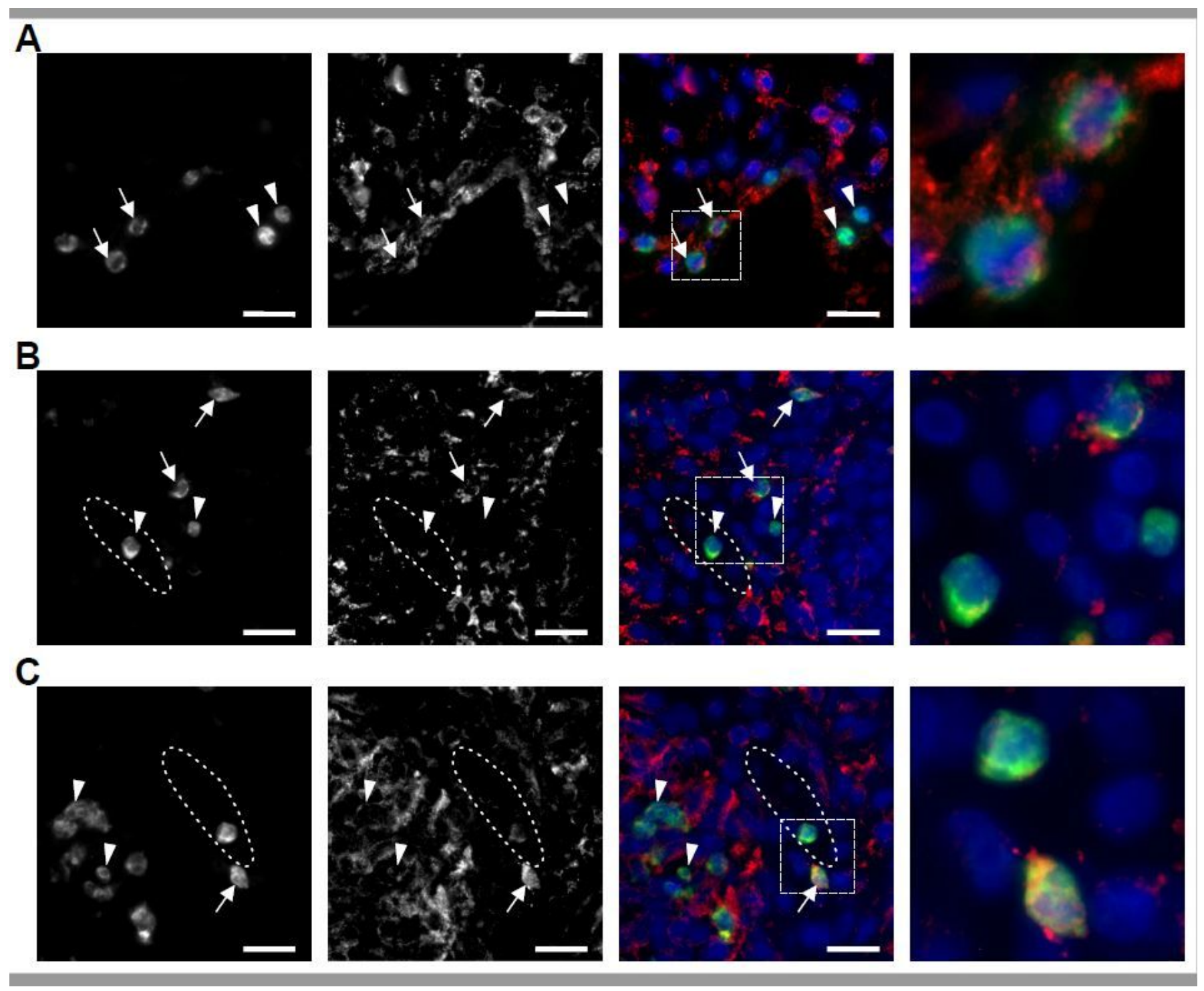

Figure 2

CD206 expressing neutrophils are found in synovial tissue. Synovial tissue biopsies from three patients, stained for MPO (green), CD206 (red) and DAPI (blue). Representative images of A) patient 4, B) patient 8, and C) patient 18. Neutrophils expressing CD206, indicated with arrows, were found in all biopsies. Neutrophils without CD206 expression, indicated with arrow heads, were found in all three biopsies. Synovial vessels are indicated with dotted ellipses. Neutrophils were found both scattered in the synovial tissue and inside synovial blood vessels. Fourth image in each panel represents a magnification of the area indicated in merged image. Scalebar is $20 \mu \mathrm{m}$. All images are taken at $40 \mathrm{X}$ magnification. 


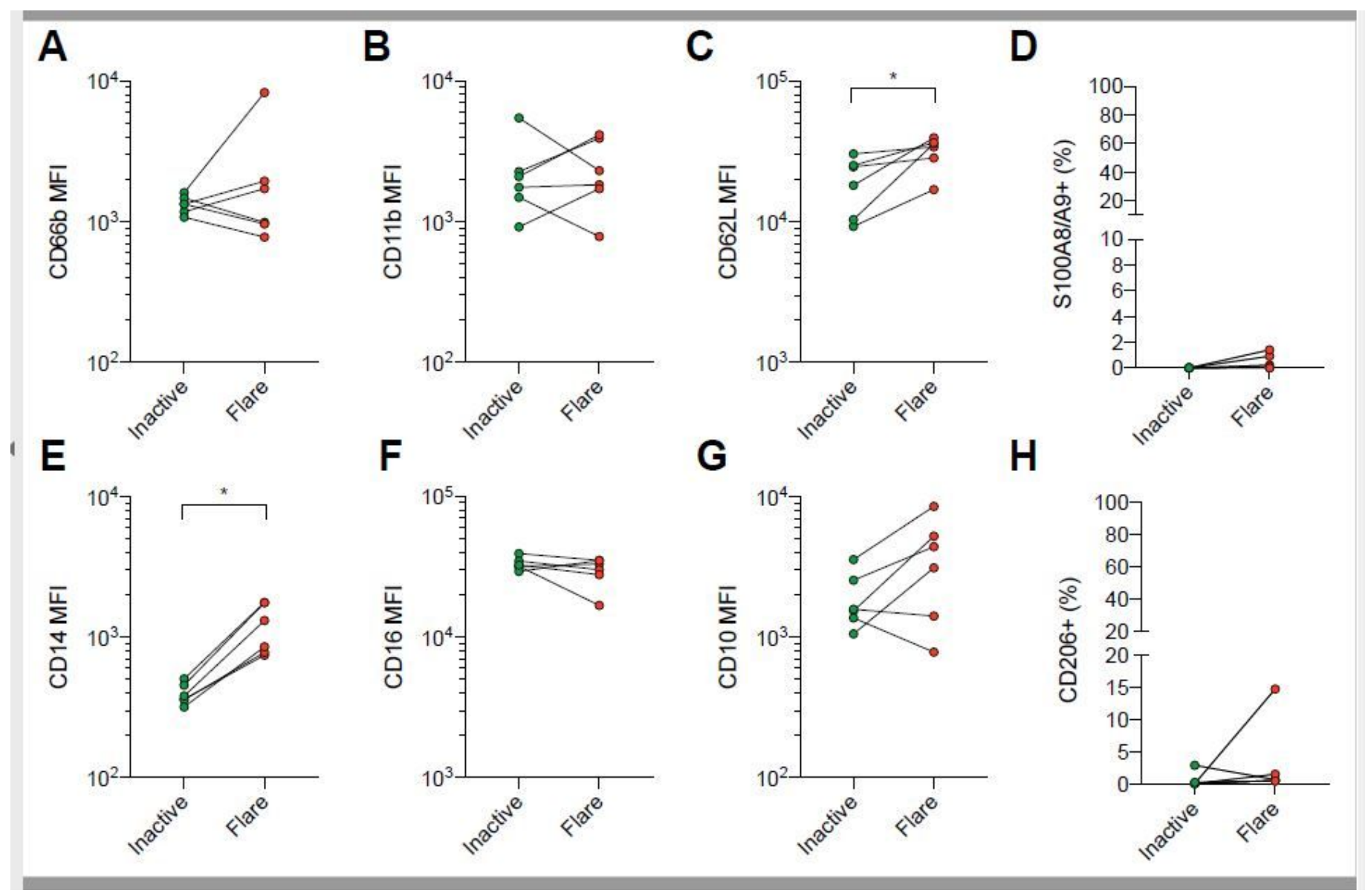

Figure 3

Neutrophil phenotypes in JIA blood are similar during flare and inactive disease. Neutrophil surface marker levels in paired blood samples taken during arthritis flare or inactive disease without arthritis. A-C \& E-G) Median fluorescence intensity (MFI) of neutrophil expression of A) CD66b, B) CD11b, C) CD62L, E) CD14, F) CD16 and G) CD10. D \& H) Proportion of neutrophils, presented as \% of total neutrophils, with cell surface expression of D) S100A8/A9 and H) CD206. * $p<0.05$, Wilcoxon signed-rank test 
B C
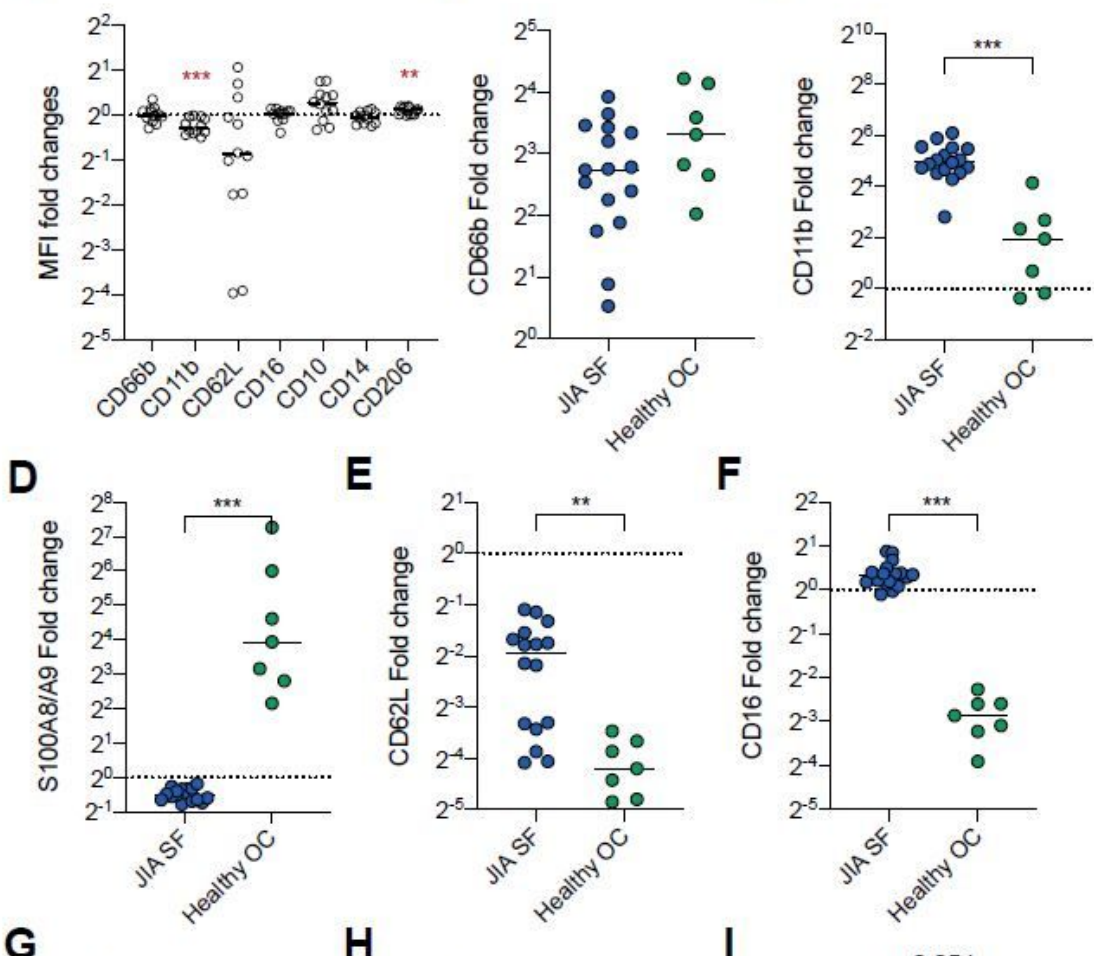

E

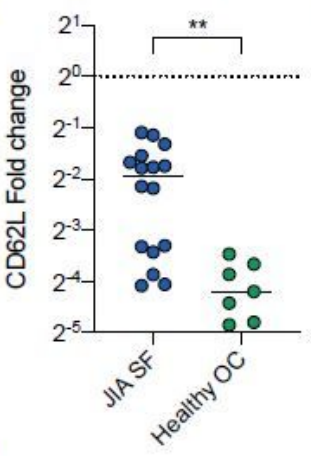

F

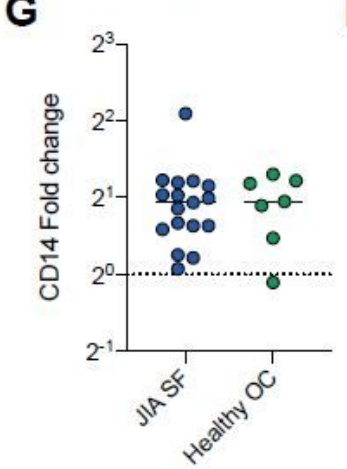

H
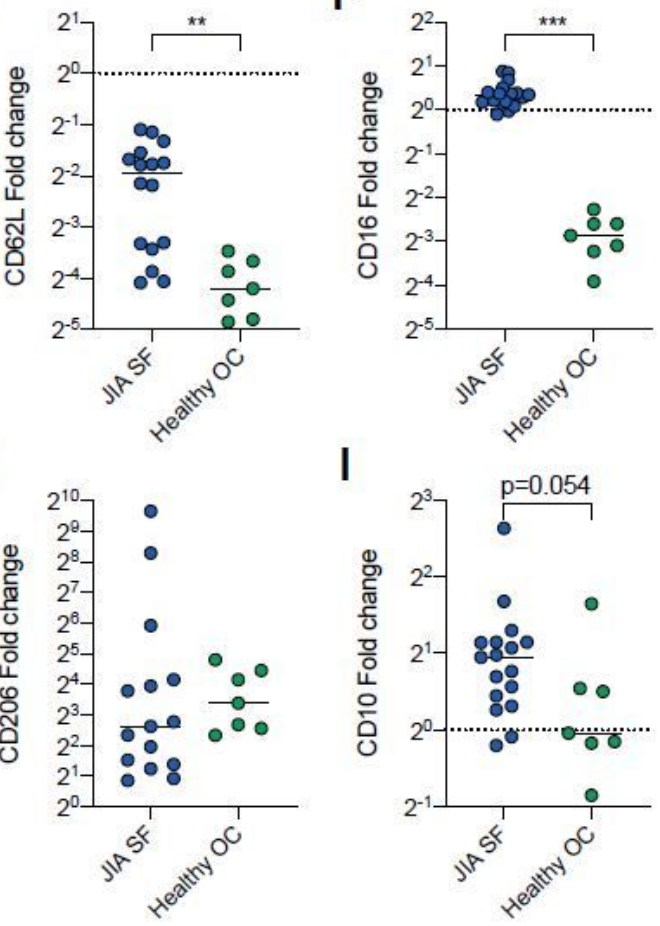

Figure 4

The neutrophil phenotype shift is not explained by exposure to synovial fluid or transmigration. A) Healthy control blood neutrophils were challenged with $20 \%$ JIA synovial fluid $(n=12)$ or $20 \%$ serum from the healthy blood donor for $1 \mathrm{~h}$ followed by analysis of surface marker expression. Control serum stimulated neutrophils are represented by the dotted line. Data are presented as fold change of median fluorescence intensity (MFI) in synovial fluid stimulated compared to control neutrophils. B-I) Neutrophils in JIA synovial fluid (SF) were compared to neutrophils from the oral cavity (OC) of healthy controls, to investigate the alterations in surface markers between blood and tissue in paired samples of blood and SF or blood and OC. Blood values are represented by the dotted line. Data are presented as fold change of MFI values of B) CD66b, C) CD11b, D) S100A8/A9, E) CD62L, F) CD16, G) CD14, H) CD206 and I) CD10 in tissue neutrophils compared to paired blood neutrophils. Line at median. ${ }^{\star \star} p<0.01,{ }^{\star \star \star} p<0.001$, Mann-Whitney U-test. 

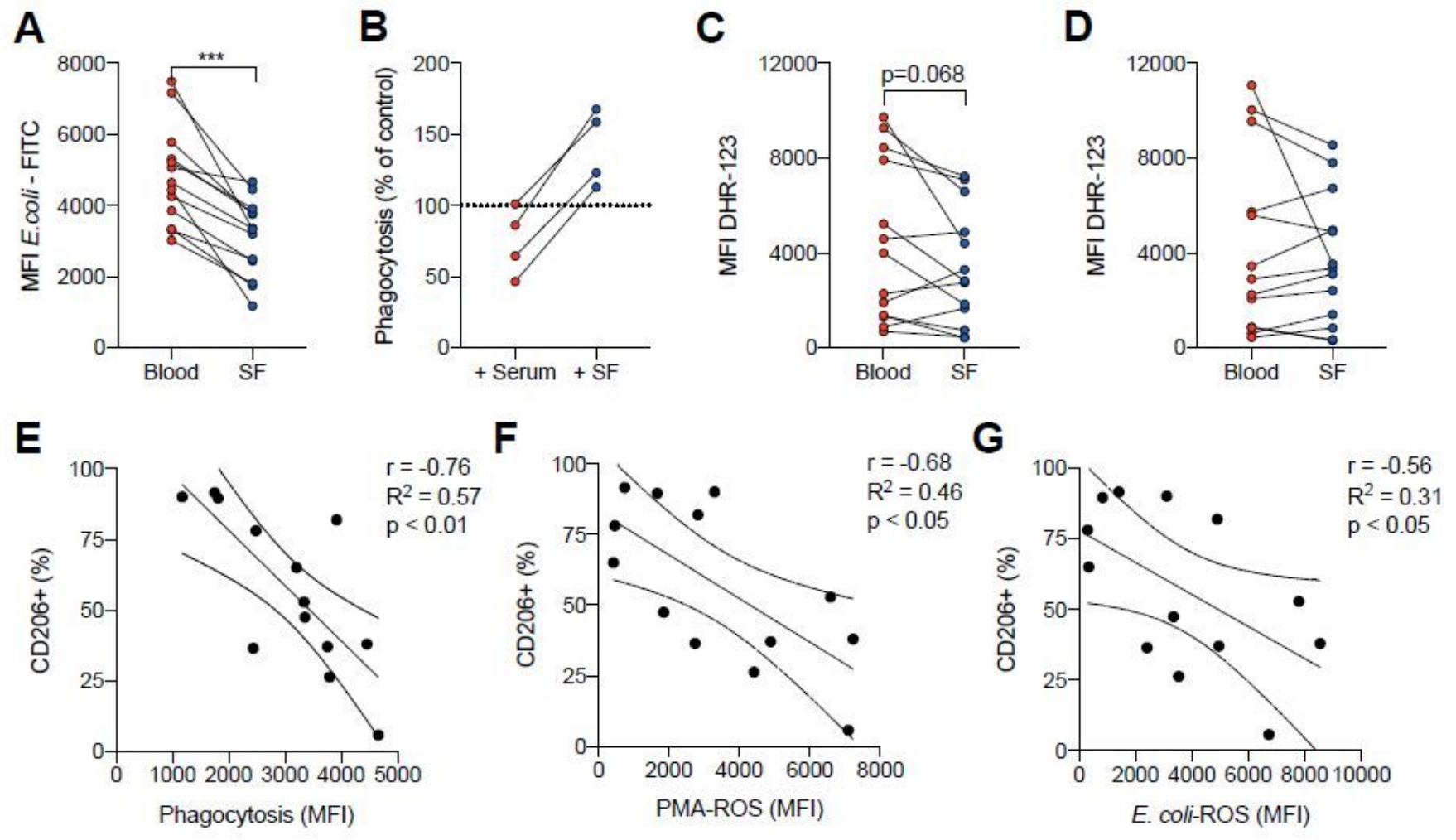

Figure 5

Neutrophil effector functions are impaired in JIA blood and synovial fluid. A) Phagocytosis of fluorescently labelled opsonized E. coli in neutrophils from paired samples of JIA blood and synovial fluid (SF). B) Phagocytosis of fluorescently labelled opsonized E. coli in healthy control neutrophils in a mixture of $10 \%$ whole blood and $90 \%$ serum or cell-free synovial fluid from four JIA patients. Results are presented as phagocytosis, measured as MFI, in serum/SF-treated samples compared to nontreated whole blood. C-D) Neutrophil ROS production, quantified using DHR-123 fluorescence, after stimulation with C) PMA or D) opsonized E. coli. E-G) Pearson r correlation analysis, with $95 \%$ confidence interval, of proportion of CD206+ neutrophils in synovial fluid with E) phagocytosis of opsonized E. coli, F) ROS production by PMA-stimulation and G) ROS production by opsonized E. coli stimulation. *** $\mathrm{p}<0.001$, Wilcoxon signed-rank test. MFI - median fluorescence intensity

\section{Supplementary Files}

This is a list of supplementary files associated with this preprint. Click to download.

- Supplementalfigures.pdf 